\title{
Stroboscopic illumination scheme for seamless 3D endoscopy
}

\author{
Neil T. Clancy*1,2 ${ }^{*}$ Danail Stoyanov ${ }^{3}$, Guang-Zhong Yang ${ }^{1,4}$ and Daniel S. Elson ${ }^{1,2}$ \\ ${ }^{1}$ Hamlyn Centre for Robotic Surgery, Imperial College London, UK; \\ ${ }^{2}$ Department of Surgery and Cancer, Imperial College London, UK; \\ ${ }^{3}$ Centre for Medical Image Computing, Department of Computer Science, University College \\ London, UK; \\ ${ }^{4}$ Department of Computing, Imperial College London, UK
}

\begin{abstract}
Intraoperative 3D imaging during minimally invasive surgery (MIS) is possible using structured lighting and has applications in the quantification of tissue morphology. However, projection schemes containing various patterns and colours can be disruptive to the surgeon's field of view. In this paper, a stroboscopic system is proposed in which structured lighting and white light images are interleaved during a high-speed camera acquisition so that the patterned light is not perceived and white light can be used solely for navigation and visual assessment. A beam chopper synchronised with the camera switches rapidly between the two lighting modes while still providing video rate display. A spectrally-encoded structured lighting system is provided by an optical fibre-based probe developed in our lab and is suitable for use in endoscopic biopsy channels. In this dual acquisition mode it is possible to display an augmented view so that the centroids of the structured lighting features are visible on the white light image. Sequential acquisition of varying exposure time images with the high speed camera also allowed the generation of high dynamic range images of the wavelength-encoded structured lighting pattern. Possible applications of this work include classification of polyp morphology as an indicator of pathology.
\end{abstract}

Keywords: Structured light, endoscopy, stroboscopic illumination, high dynamic range

\section{INTRODUCTION}

Tissue surface shape is of clinical interest as tissue morphology may be an indicator of pathology. For example, visual inspection of colonic polyps have revealed correlations between their shape and whether or not they were neoplastic ${ }^{1}$. Analysis of this shape in a quantitative manner using a shape-based classification scheme could aid biopsy site selection and decrease the detection of false positives and negatives.

Quantification of tissue shape also has potential applications in MIS, where it could be employed to aid registration of pre-operative images, such as MRI, US or CT, with the live surgical view. A real-time updated 3D mesh of the tissue in the field of view could form a deformable framework onto which a 3D scan of a tumour could be overlaid, forming an augmented reality view ${ }^{2,3}$ to aid demarcation of tumour margins.

Both the tissue inspection and image registration applications mentioned thus far are achieved endoscopically, where the small cross-sectional area of the instruments used limits the space available to a 3D imaging device. Furthermore, for inspection of colonic polyps, a flexible endoscope must be used, which puts additional demands on the size of the optics and their mechanical properties. A recent publication by our group has addressed these demands and presents a miniature fibre-based structured lighting probe with gradient refractive index optics within a maximum outer diameter of $1.9 \mathrm{~mm}^{4}$. The probe is small enough to fit into an endoscopic biopsy port and the illumination source is a supercontinuum laser with relatively high optical throughput $(>100 \mathrm{~mW})$ at the distal end to create a pattern that is easily detected by the camera. The probe uses spectral encoding in the visible range to label each projected feature so that it may be identified during the $3 \mathrm{D}$ triangulation process.

However, due to the fact that the pattern used is made up of multiple colours across the visible range from approximately $450-650 \mathrm{~nm}$, normal navigation and visualization becomes confusing. One approach to this problem that has been used in non-biological scenarios is the technique of 'imperceptible structured light', which involves the

*n.clancy@imperial.ac.uk; imperial.ac.uk/roboticsurgery

Advanced Biomedical and Clinical Diagnostic Systems X, edited by Tuan Vo-Dinh,

Anita Mahadevan-Jansen, Warren S. Grundfest, Proc. of SPIE Vol. 8214, 82140M - (C) 2012 SPIE

CCC code: $1605-7422 / 12 / \$ 18 \cdot$ doi: $10.1117 / 12.909360$

Proc. of SPIE Vol. $821482140 \mathrm{M}-1$ 
alternate projection of two complementary patterns at a high framerate ${ }^{5}$. These patterns may be simple stripes ${ }^{6}$ or more complex ${ }^{7}$. If the projection frequency is high enough, the human eye will only perceive one evenly illuminated area rather than two distinct patterns. To preserve the colour information in the scene being imaged however, the sum of all of the coloured patterns must be white light for all pixels ${ }^{8}$, which is not the case with the miniature multispectral structured lighting probe described earlier.

In this paper, an interleaved dual illumination scheme is proposed where a beam chopper is used to alternately switch between white and structured light with endoscope-compatible optics. The measured frequency of the high precision chopper is used to trigger a high speed camera so that the computer display shows the scene as illuminated under white light, with the images under patterned light saved for segmentation and 3D processing. This stroboscopic system allows video rate display $(15 \mathrm{~Hz})$ at high resolution $(1024 \times 1024$ pixels $)$.

Although the labels assigned to the projected features in the wavelength-based segmentation are insensitive to the background optical properties, they may still be affected by varying reflectivity by becoming more or less intense on different surfaces. During surgery the reflectivity of tissue may change sharply between different tissue types, for example, white highly reflecting fat and dark highly absorbing liver. Since the projected pattern works in the visible range of the spectrum, different spots will be visible to varying degrees: blue being absorbed strongly by haemoglobin in comparison to red. This, coupled with the fact that the emission strength of the laser is weaker at the blue end, means that for any given surgical scene not all of the projected features will be clearly visible. In this paper, a high speed camera allows the acquisition of a number of frames of varying exposure time so that a 'high dynamic range' image can be created where less bright blue dots are correctly exposed without saturating the red ones.

\section{MATERIALS AND METHODS}

\subsection{Multispectral Structured Lighting Probe}

The structured lighting probe used in this paper has been described in detail elsewhere ${ }^{4}$ but a brief description is provided here. The broadband output of a supercontinuum laser is directed onto a prism which disperses the light into its constituent wavelengths. This dispersed light is then focused onto a linear array of $12750 \mu \mathrm{m}$ core fibres at the proximal end of the fibre probe. These fibres are bundled incoherently and are packed into a circular arrangement at the distal end so that the result is a mixing of the laser wavelengths and a different wavelength per fibre, each with a full-width at halfmaximum of approximately $5 \mathrm{~nm}$. Finally, a GRIN lens is used to form a sharp image of the probe's end face over working distances from less than $2 \mathrm{~cm}$ to $20 \mathrm{~cm}$. The colour camera interprets each of these spots, which effectively have a pure wavelength, as varying amounts of red, green and blue. Converting the RGB space to chromaticity coordinates allows calculation of the dominant wavelength detected at a particular pixel, which is independent of the background colour due to its narrow bandwidth. The calculated wavelength of a particular spot then serves as its label so that it may be identified and its position triangulated in 3D space using active stereo techniques used previously ${ }^{9,10}$.

\subsection{Stroboscopic illumination}

In this paper, a high speed camera synchronized with a stroboscopic illumination system is proposed that separates the white light and structured lighting sources onto alternate frames. The system is designed so that the white light frames are displayed live and the structured lighting images are saved for further processing and 3D triangulation.

A schematic of the system in Figure 1 shows how an optical beam chopper (3501 Optical Chopper, New Focus, Inc., USA) is used to stroboscopically switch between white light and laser illumination. Both beam paths are directed through the chopper wheel so that they are out of phase by $90^{\circ}$ and the chopping frequency is outputted as a square wave voltage signal $(0-5 \mathrm{~V})$. The laser output is collimated and so is chopped as it propagates in free space. The xenon light (xenon 300, Karl Storz GmbH, Germany) is first coupled into a light cable (5 mm diameter optical fibre bundle; Karl Storz GmbH, Germany) which is then placed as close as possible to the beam chopper, with a second light cable on the other side. This can then be connected to the standard lighting input of a laparoscope or flexible endoscope. The chopping signal is then used to trigger a high-speed colour camera (Prosilica GX1050c, Allied Vision Technologies, Inc., USA).

The camera streams colour image data via two gigabit ethernet cables, enabling a maximum speed of $112 \mathrm{fps}$. The acquisition sequence is detailed in Figure 2. On the rising edge of the trigger signal $\left(\mathrm{t}_{1}\right)$, white light is allowed through the chopper and displayed on screen. On the falling edge $\left(\mathrm{t}_{2}\right)$, laser light is transmitted and an image of the structured lighting pattern is saved for wavelength-based segmentation and 3D triangulation. 


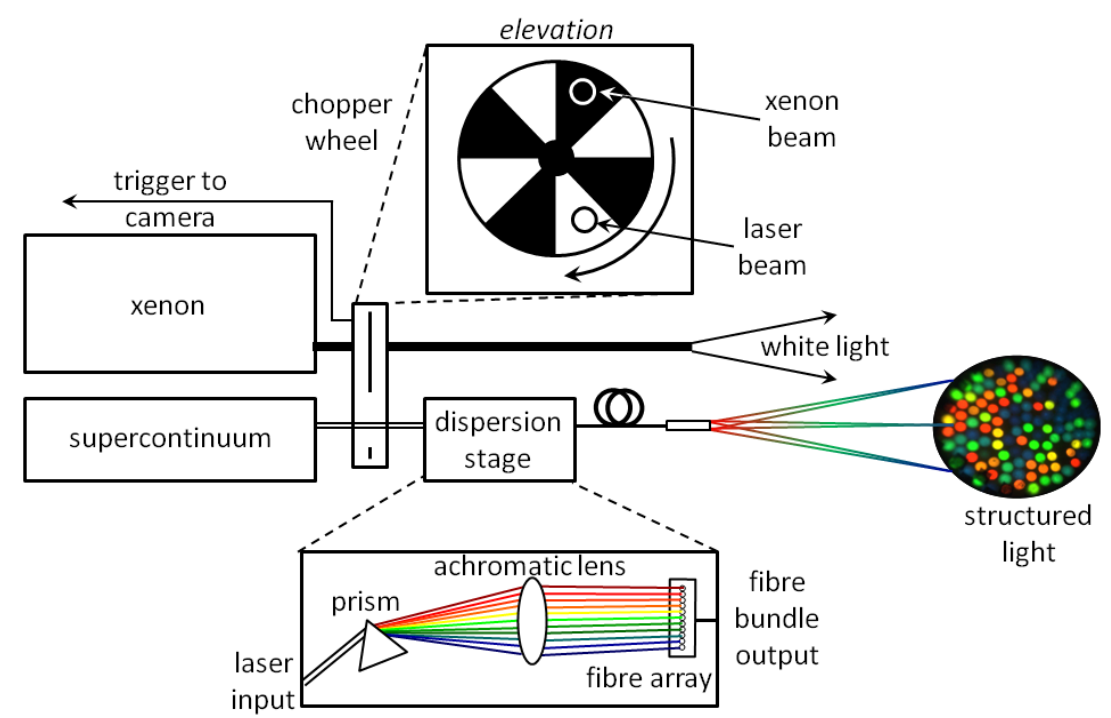

Figure 1. Stroboscopic illumination system. The optical chopper is placed in both white light and laser beam paths so that they are alternately switched on and off.

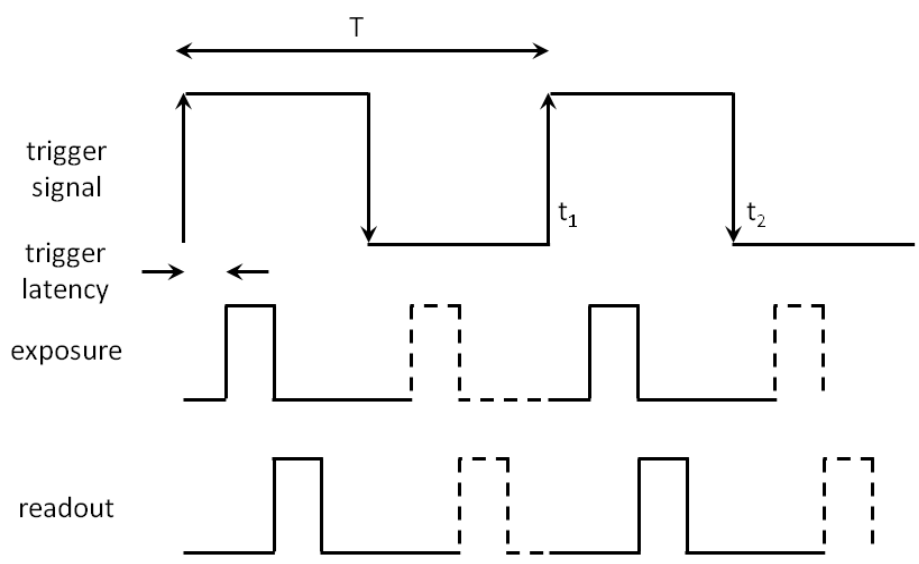

Figure 2. Trigger timing schematic. The $5 \mathrm{~V}$ output signal from the chopper wheel is used to trigger acquisitions from the camera at both the rising and falling edges, $t_{1}$ and $t_{2}$ respectively. The chopper period is indicated by $T$, while the trigger latency, exposure and readout times define the upper limit of the acquisition framerate.

The final framerate of the system is dependent on the exposure time needed, trigger latency period and the readout time, which is itself determined by the image height. According to the manufacturer's specifications, the trigger latency is approximately $1.5 \pm 0.5 \mu \mathrm{s}$ and for an image height of 1024 pixels, a framerate of $112 \mathrm{~Hz}$ is possible in free run mode. The relationship between image height and maximum framerate is approximately a negative exponential, with $200 \mathrm{~Hz}$ possible at an image height of 400 pixels.

\subsection{High dynamic range imaging}

The multispectral structured lighting probe generates a pattern of dots that are assigned unique wavelength labels. To form a high dynamic range image of the dot pattern with all colours correctly exposed, a number of low dynamic range images were acquired with varying exposure times. In this approach, RGB pixels in one particular image in the sequence where saturation or low intensity values are detected are replaced by pixels from an image of more suitable exposure time. To demonstrate the procedure, a sample of ex vivo tissue (lamb's liver) was used as the target illuminated by the structured lighting pattern. Two successive images were then acquired with exposure times of $1.3 \mathrm{~ms}$ and $4.0 \mathrm{~ms}$. The high dynamic range image was created by combining these images using an algorithm in Matlab's 'Image Processing Toolbox' (The Math Works, Inc., USA). The low exposure time image was used as a base image and its pixel values checked for agreement with the noise and saturation settings. Any pixel with a grayscale value below 10 was considered 
too noisy and replaced by the corresponding RGB triplet from the longer exposure image, while any saturated pixel (grayscale level greater than 250 in either the red, green or blue colour planes) was not included in the final image. The algorithm then smoothed the boundaries of the resulting image by solving Laplace's equation to remove discontinuities.

\section{RESULTS}

\subsection{Stroboscopic illumination}

Figure 3 shows the output from the stroboscopic system. White light illuminated frames provide a clear view of the tissue to the surgeon, while the structured lighting pattern is saved in the background. These images can then be used to reconstruct the surface shape of the tissue in view by finding the centroids of each spot using the wavelength-based segmentation algorithm and triangulating their $3 \mathrm{D}$ coordinates.
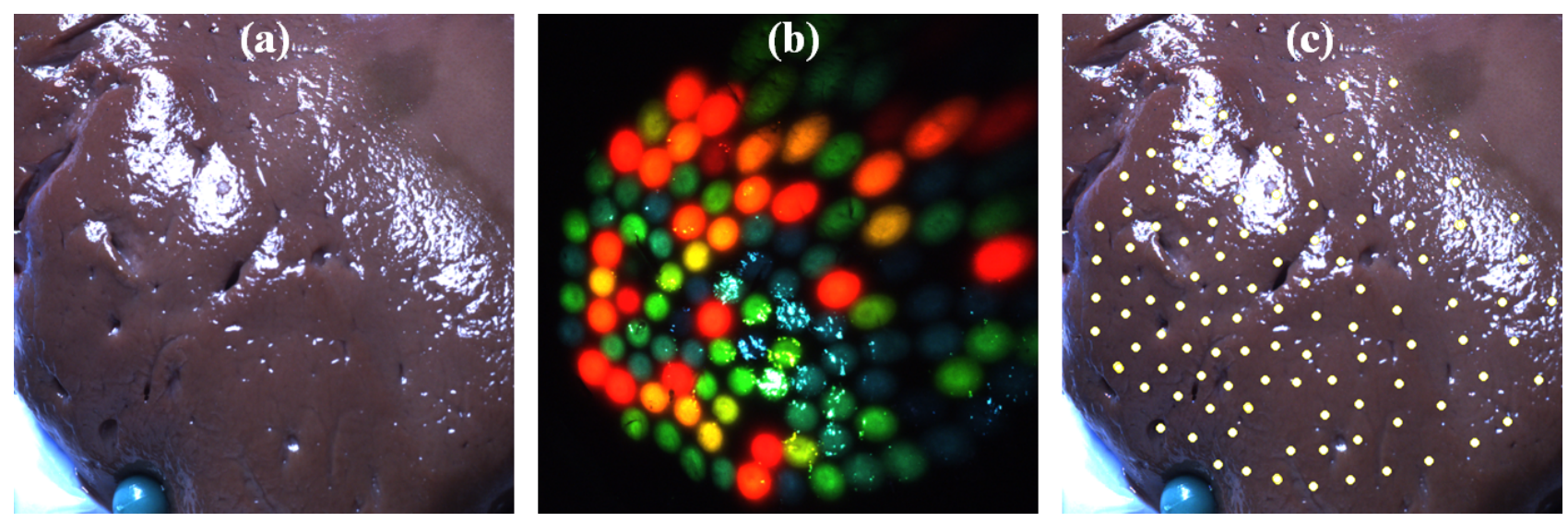

Figure 3. Synchronised stroboscopic imaging of liver ex vivo showing (a) the white light image displayed on screen, (b) the structured lighting image saved to disk and (c) the pattern centroids computed using the structured lighting algorithm ${ }^{4}$ superimposed on the white light image.

After the projected spot coordinates were obtained, they could be overlaid onto the white light image, indicating the region covered by the structured lighting pattern to the surgeon without the distracting presence of the varying spot colour.

\subsection{High dynamic range imaging}

To maintain sufficient image brightness without saturating pixels, two images of different exposure times were acquired using the high speed camera. At an exposure time of $1.3 \mathrm{~ms}$, Figure 4 (a) shows that the red dots are correctly resolved and but the green and blue dots are much more dim, and are barely visible above the noise. When the exposure time is increased to $4 \mathrm{~ms}$, as in Figure 4 (b), these dots become visible but the red dots are now saturated. This overexposure has the effect of making these dots more difficult to distinguish from each other and, as a result, will introduce errors into the 3D reconstruction. Figure 4 (c) shows the high dynamic range image created by using the most desirable pixels from each image, subject to the inclusion criteria.

This is illustrated in Figures 4 (d) to (f) where the grayscale profiles across two sample spots are presented for the two low dynamic range images and the high dynamic range image. In Figure 4 (d) the blue dot has a maximum grayscale value that is just above the background noise, while the red spot signal is much stronger and occupies more of the grayscale range. When the exposure time is increased, as in Figure 4 (e), the blue spot is almost four times brighter than it was initially, but the red is now saturated. Since the blue and green also contribute to the colour of this red spot however, erroneous wavelength values will be calculated in the segmentation algorithm as the ratio of red to green and blue is no longer constant across its diameter. The high dynamic range profile in Figure 4 (f) combines the two images so that the blue spot is brighter and the red spot is not saturated and is correctly in proportion to its green and blue components. 

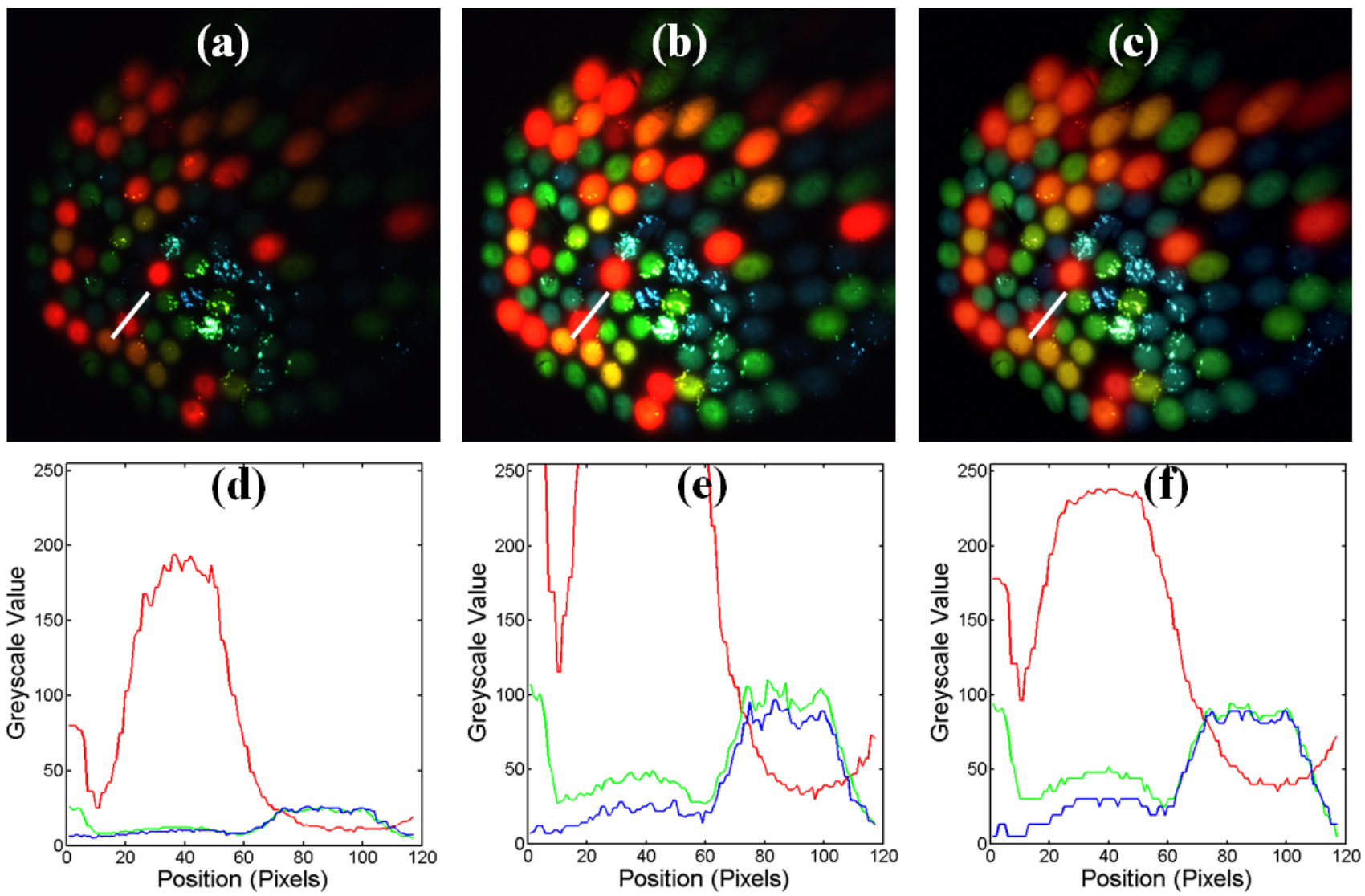

Figure 4. High dynamic range imaging of ex vivo liver tissue. (a) Exposure time $=1.3 \mathrm{~ms}$; red correctly exposed, blue and green noisy. (b) Exposure time $=4 \mathrm{~ms}$; blue and green visible, red overexposed. (c) High dynamic range image; red, green and blue spots visible without saturation. (d)-(f) Grayscale profiles corresponding to the values along the white lines in (a)-(c).

\section{DISCUSSION AND CONCLUSIONS}

A stroboscopic illumination system for imperceptible structured lighting during MIS has been demonstrated. At full resolution (1024×1024 pixels), it is possible to display white light illuminated frames at $15 \mathrm{~Hz}$ while saving structured lighting images for further processing. Using a previously developed segmentation algorithm, the different wavelength spots in the pattern were segmented from the background tissue and their centroids calculated. These can then be used as the input to the $3 \mathrm{D}$ reconstruction algorithm that triangulates their position. The dual acquisition mode of the system allows the user to overlay the centroids on the white light image with minimal disruption to normal viewing. This is particularly advantageous as it provides the surgeon with fine control over the pattern illumination area and allows checking for the presence of occluding tissue and whether or not the correct tissue is being measured.

The high speed camera has also been shown to be capable of acquiring multiple images to generate a high dynamic range image of the projected multispectral pattern in order to correct for high absorption by blood in the blue and green range of the spectrum. The result is an image that is low-noise with features that are more easily segmented than would otherwise be possible in a single exposure low dynamic range image. A limitation of this method is that the low dynamic range images used must be spatially aligned. Therefore, any movement of the tissue must be small in comparison to the acquisition time of the image set. At full resolution, this system is capable of saving images at $15 \mathrm{~Hz}$, which is potentially quick enough to image the abdomen and bowel as proposed, where small movements related to breathing and peristalsis are present. However, a higher framerate may be necessary to avoid misregistration during sharp endoscope camera movements or faster moving tissue such as the beating heart.

The stroboscopic system described here displays white light images and saves structured lighting images for offline processing. Future work will involve optimization of the acquisition software to allow online simultaneous processing of the structured lighting data without the need for saving every image. The stability of the high dynamic range images will 
also be investigated to validate the robustness of the projected feature segmentation after combination of the multiple low dynamic range images. Adjustment of the beam chopper duty cycle will enable the integration of the high dynamic range images and white light display with a decreased risk of misalignment errors. For example, a $75 \%$ duty cycle, rather than the $50 \%$ described here, would allow the successive acquisition of three structured light images before being interrupted by a white light image. Finally, the white light illumination side of the system could be made to be more efficient as the current air gap proximity coupling loses a lot of light due to reflection alone. Use of an ultra-bright LED source may be a viable alternative as it may be switched on and off electronically, removing the need for it to interact with the chopper.

\section{ACKNOWLEDGEMENTS}

Funding for this work was provided by ERC grant 242991, and UK EPSRC and Technology Strategy Board grants EP/E06342X/1 and DT/E011101/1. Danail Stoyanov would like to acknowledge the financial support of a Royal Academy of Engineering/EPSRC Fellowship.

\section{REFERENCES}

[1] Kato, S., Fu, K. I., Sano, Y., Fujii, T., Saito, Y., Matsuda, T., Koba, I., Yoshida, S., and Fujimori, T., "Magnifying colonoscopy as a non-biopsy technique for differential diagnosis of non-neoplastic and neoplastic lesions," World J. Gastroenterol., 12(9), 1416-1420 (2006).

[2] Stoyanov, D., Mylonas, G. P., Lerotic, M., Chung, A. J., and Yang, G.-Z., "Intra-operative visualizations: perceptual fidelity and human factors," J. Disp. Technol., 4(4), 491-501 (2008).

[3] Edwards, P. J., King, A. P., Hawkes, D. J., Fleig, O., Maurer, C. R. J., Hill, D. L., Fenlon, M. R., de Cunha, D. A., Gaston, R. P., Chandra, S., Mannss, J., Strong, A. J., Gleeson, M. J., and Cox, T. C., "Stereo augmented reality in the surgical microscope," Stud. Health Technol. Inform., 62, 102-108 (1999).

[4] Clancy, N. T., Stoyanov, D., Maier-Hein, L., Groch, A., Yang, G.-Z., and Elson, D. S., "Spectrally-encoded fibre-based structured lighting probe for intraoperative 3D imaging," Biomed. Opt. Express, 2(11), 3119-3128 (2011).

[5] Raskar, R., Welch, G., Cutts, M., Lake, A., Stesin, L., and Fuchs, H., "The office of the future: a unified approach to image-based modeling and spatially immersive displays," Proceedings of ACM SIGGRAPH. 179188 (1998).

[6] Fuchs, H., Cotting, D., Naef, M., and Gross, M., [Methods, systems, and computer program products for imperceptibly embedding structured light patterns in projected color images for display on planar and nonplanar surfaces] The University of North Carolina, USA, Patent No. US 7,182,465 B2 (2007).

[7] Molinier, T., Fofi, D., Salvi, J., and Gorria, P., "2D virtual texture on 3D real object with coded structured light," Proc. of SPIE-IS\&T Electronic Imaging. 6813, 68130Q (2008).

[8] Dai, J., and Chung, R., "Head pose estimation by imperceptible structured light sensing," International Conference on Robotics and Automation. 1646-1651 (2011).

[9] $\mathrm{Wu}, \mathrm{T} . \mathrm{T}$. , and $\mathrm{Qu}$, J. Y., "Optical imaging for medical diagnosis based on active stereo vision and motion tracking," Opt. Express, 15(16), 10421-10426 (2007).

[10]Batlle, J., Mouaddib, E., and Salvi, J., "Recent progress in coded structured light as a technique to solve the correspondence problem: a survey," Pattern Recogn., 31(7), 963-982 (1998). 